\title{
Nuevos datos sobre Althos distinctus (Signoret, 1864) (Hemiptera: Heteroptera: Coreidae) en Patagonia
}

\author{
New data on Althos distinctus (Signoret, 1864) \\ (Hemiptera: Heteroptera: Coreidae) from Patagonia
}

Eduardo I. Faúndez ${ }^{1} \&$ Flavia Raffo ${ }^{2}$

Coreidae Leach, 1815 es una numerosa familia de heterópteros, que incluye unas 2550 especies clasificadas en cerca de 436 géneros (Faúndez, 2015). Los coréidos son principalmente fitófagos; sin embargo se les ha registrado consumiendo carroña e incluso infringiendo picaduras a seres humanos (Faúndez \& Carvajal, 2011). Muchas especies han sido consideradas de una alta importancia económica, por el daño que producen especialmente en los frutos de muchos cultivos (Mitchell, 2000).

En la Patagonia son escasos los datos existentes sobre esta familia (Diez et al. 2016; Faúndez, 2016), e incluso la situación de algunas especies ha permanecido sin revisar por un largo periodo (Faúndez \& Carvajal, 2016).

Althos distinctus (Signoret, 1864) (Fig. 1) es un coréido de amplia distribución en Chile, abarcando desde la región de Valparaíso hasta Aysén, sin localidad específica (Brailovsky, 1990). En Argentina se le ha citado recientemente de Bariloche, provincia de Río Negro (Olivera et al. 2017). Adicionalmente, hasta el momento se desconocen sus limites septentrionales de distribución y datos sobre la biología de esta especie. Por lo tanto, el objetivo de esta contribución es entregar nuevos registros que aportan datos sobre su distribución y biología en la Patagonia.

Material examinado: Argentina: Provincia de Santa Cruz, Parque Nacional los Glaciares, 12-I-1985, sobre Senecio sp. J. Ulloa leg. $10^{\top}$ (E. Faúndez coll.); I-2017, El Calafate,

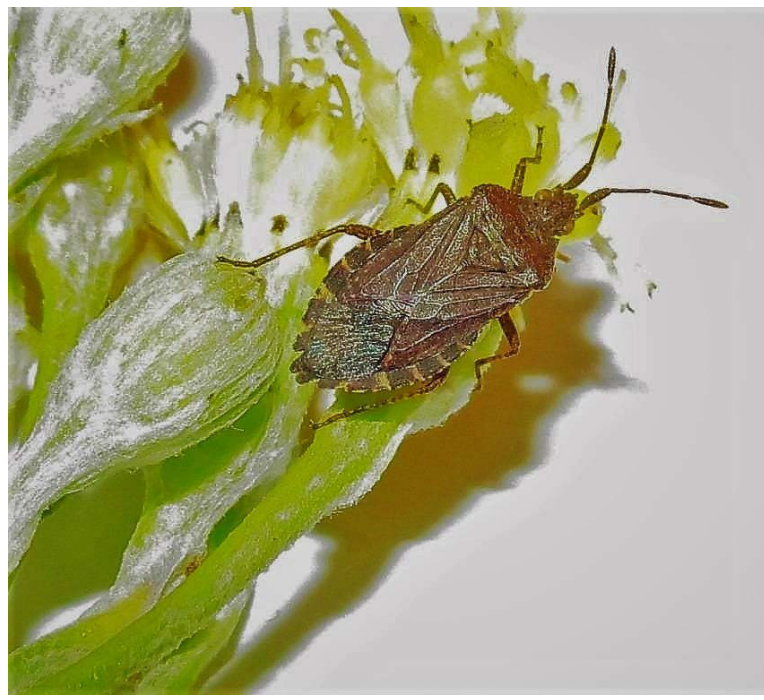

Fig. 1. Hembra de Althos distinctus sobre Senecio patagonicus en El Calafate, Santa Cruz, Argentina.

50²0'16'S-72 15'53'O sobre Senecio patagonicus Hook et Arn. F. Raffo leg.19 (Fig. 1) (F. Raffo coll.). CHILE: Región de Los Lagos, P. N. Puyehue, 16-I-1979, on Senecio sp., moist vegetation, pond marginal. Ashworth \& Hoganson leg. $10^{\top 1}$ (E. Faúndez coll.); Región de Aysén, Coyhaique, Balmaceda, 4542'25'S-7141'33'O,

Instituto de la Patagonia, Universidad de Magallanes, Av. Bulnes 01855, Punta Arenas, Chile.

$\varangle$ ed.faundez@gmail.com

2 Parque Nacional Los Glaciares, El Calafate,

Av. del Libertador 1302.CP 9405. Santa Cruz. Argentina. 
10-II-2013, 525 m s.n.m., 2o10, A. Lüer leg. (E. Faúndez, coll.).

Los registros aquí entregados extienden la distribución de esta especie hasta la provincia de Santa Cruz en Argentina y el registro de Coyhaique es la primera localidad específica documentada para la región de Aysén en Chile. El Calafate es la localidad más septentrional conocida para esta especie, sin que hasta la fecha se haya registrado en Magallanes o Tierra del Fuego (Faúndez, 2016). Adicionalmente, los registros en Senecio sp. y Senecio patagonicus (Asteraceae) son los primeros de una planta hospedadora para esta especie. Brailovsky (1990) indica para algunas especies de Althos del norte de Sudamérica y Centroamérica y Norteamérica algunas asteráceas como hospedadoras, sin que hasta el momento haya registros en las especies más australes. Hasta el momento, no se han registrado especies de Althos como causantes de daño económico, sin embargo dada la complejidad del grupo en muchas ocasiones son solamente considerados como Coreidae, por lo que, sus interacciones con los cultivos, son una arista que aún resta por imvestigar. Dado que esta es una de las especies con distribución más septentrional, es posible que la asociación de este género con las asteráceas se mantenga a lo largo de todo su rango de distribución; se ha registrado que estos insectos recurren a otras plantas para completar sus requerimientos nutritivos, en casos de escasez alimenticia, tal como ha sido indicado para varios integrantes de esta familia (Faúndez, 2017). Por esto mismo, consideramos posible que esta especie alcance el norte de la región de Magallanes incluyendo parte de Tierra del Fuego, ya que representantes de Senecio (incluyendo $S$. patagonicus) se encuentran en estas latitutdes, pero que probablemente no ha sido colectada por faltas de muestreos en la época adecuada del año, ya que sus poblaciones aparentan ser escasas $y$ muy estacionales.

\section{LITERATURA CITADA}

Brailovsky, H. (1990). Revisión del complejo "Althos" (Hemiptera - Heteroptera -
Coreidae). Publicaciones Especiales del Instituto de Biología, Universidad Nacional Autónoma de México, 5, 1-156.

Diez, F., Ruiz Espindola, M., Cornelis, M., \& Coscarón, M. C. (2016). Additional information for Leptoglossus impictus (Stål, 1859) (Hemiptera: Heteroptera: Coreidae: Coreinae: Anisoscelini) from Patagonia, Argentina. Zootaxa, 4067(4), 494-500.

Faúndez, E. I. (2015). Synopsis of the genus Eldarca Signoret, 1864 (Hemiptera: Heteroptera: Coreidae). Zootaxa, 3915(2), 287-294.

Faúndez, E. I. (2016). The Coreoidea Leach, 1815 (Hemiptera: Heteroptera) of Magallanes Region: Checklist and identification key to the species. Anales del Instituto de la Patagonia, 44(1), 39-42.

Faúndez, E. I. (2017). Comentarios y correcciones sobre la familia Coreidae Leach, 1815 (Hemiptera: Heteroptera) en libros chilenos. Arquivos Entomolóxicos, 18, 17-22.

Faúndez, E. I., \& Carvajal, M. A. (2011). Primer registro de una picadura de Leptoglossus chilensis (Spinola, 1852) (Hemiptera: Heteroptera: Coreidae) en un ser humano. Boletín de Biodiversidad de Chile, 6, 2225.

Faúndez, E. I., \& Carvajal M. A. (2016). Sobre la relación entre Leptoglossus chilensis (Spinola, 1852) y Leptoglossus concaviusculus Berg, 1892 stat. rest. (Heteroptera: Coreidae), con nuevos datos acerca de su morfología. Anales del instituto de la Patagonia, 44(3), 65-70.

Mitchell, P. L. (2000). Leaf-footed bugs (Coreidae) In Schaefer C. W. \& Panizzi A. R. (Eds.), Heteroptera of Economic Importance (pp. 337-403) Boca Raton, CRC Press.

Olivera, L., Melo, M. C., \& Dellapé, P. M. (2017). First records of leaf-footed bugs (Heteroptera: Coreoidea: Coreidae) from Argentina. Revista de la Sociedad Entomológica Argentina, 76(1-2), 68-70. 